Ultrastructural features (bulges) of membrane nanotubes between cone-like photoreceptor cells: An investigation employing scanning electron microscopy

Scholkmann, Felix ; Seidel, Deike ; Funk, Richard H W ; Roehlecke, Cora

DOI: https://doi.org/10.19185/matters.201802000011

Posted at the Zurich Open Repository and Archive, University of Zurich

ZORA URL: https://doi.org/10.5167/uzh-161849

Journal Article

Published Version

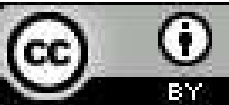

The following work is licensed under a Creative Commons: Attribution 4.0 International (CC BY 4.0) License.

Originally published at:

Scholkmann, Felix; Seidel, Deike; Funk, Richard H W; Roehlecke, Cora (2018). Ultrastructural features (bulges) of membrane nanotubes between cone-like photoreceptor cells: An investigation employing scanning electron microscopy. Matters:1-6.

DOI: https://doi.org/10.19185/matters.201802000011 


\section{Ultrastructural features (bulges) of membrane nanotubes between cone-like photoreceptor cells: An investigation employing scanning electron microscopy}

$\checkmark$ Correspondence Felix.Scholkmann@usz.ch

8 Disciplines Cell Biology

Q Keywords Membrane Nanotubes Tunnelling Nanotubes Ultrastructure

it Type of Observation Standalone

\& Type of Link

Standard Data

(-) Submitted Feb 1, 2018 (๑) Published Mar 7, 2018

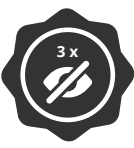

Triple Blind Peer Review The handling editor, the reviewers, and the authors are all blinded during the review process.

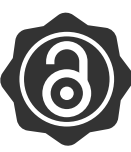

Full Open Access Supported by the Velux Foundation, the University of Zurich, and the EPFL School of Life Sciences.

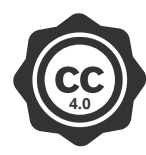

Creative Commons 4.0 This observation is distributed under the terms of the Creative Commons Attribution 4.0 International License.

\author{
Felix Scholkmann, Deike Seidel, Richard H.W. Funk, Cora Roehlecke \\ Biomedical Optics Research Laboratory, Department of Neonatology, University Hospital Zurich, University of Zurich, \\ Zurich, Research Office for Complex Physical and Biological Systems; TU Dresden, Faculty of Medicine, Institute of Anatomy
}

\begin{abstract}
Membrane nanotubes (MNTs) are nanotubular cell-to-cell connections enabling cell-tocell signaling and cargo transfer. The presence of local MNT bulges has been reported by several studies. However, a detailed characterization of MNT bulges concerning their geometrical properties has not been done yet. The aim of our study was to analyze MNT from cone-like photoreceptor cells $(661 \mathrm{~W})$ using scanning electron microscopy (SEM) in order to characterize MNT bulges, thereby increasing the knowledge of ultrastructural feature of MNTs. Our SEM analysis of two MNTs with multiple bulges revealed that (i) MNT bulges are characterized by a statistically significant local increase in the MNT diameter (125\% and $250 \%$ for both MNTs analyzed), (ii) the thickness and length of the MNT bulges correspond to dimensions of mitochondria, peroxisomes and exosomes, and (iii) the MNT bulges seem not to be randomly distributed on the MNTs but exhibit a preferred spacing with a different median value for each MNT. Our findings highlight that the ultrastructure of MNT exhibits interesting properties that need to be further investigated.
\end{abstract}

\section{Introduction}

Nanotubular cell-to-cell connections termed "tunnelling nanotubes" or "membrane nanotubes" (MNTs) (the term used in this paper) enable diverse possibilities of cell-to-cell signaling and cargo transfer [1] [2] [3]. This includes the exchange of signal carriers (e.g. proteins), organelles (e.g. mitochondria), bacteria, viruses, exosomes, DNA, RNA, or electric long-range coupling [4] [5] [6] [7] [8].

The defining characteristics of MNTs are still being debated and the investigation of MNT properties is ongoing but recently a consensus of leading MNT researchers was published that MNTs can be defined as "tubular membrane connection between non-adjacent cells that allow direct intercellular communication, not necessarily gap junction-mediated". MNTs also contain F-actin and/or tubulin, have a variable diameter of 50-800 $\mathrm{nm}$ and are open-ended [3]. It can be added that MNTs are filled with cytoplasm and have a lipid bilayer [9]. Some MNTs also contain microtubules [10] and have the gap junction protein $\mathrm{Cx} 43$ at the end [6]. In some cases, individual MNTs stick together to form a single, thicker, MNT [9].

As recently summarized by Rustom [11], MNTs are "intimately linked to the physiological state and pathological" state of cells and "represent a central joint element of diverse diseases, such as neurodegenerative disorders, diabetes or cancer". Furthermore, MNTs seem to play an important role in long-range physical cell-to-cell signaling in multicellular organisms possibly enabling novel ways of physical signal transfer [12] and being involved in the functioning of neurosystems [13].

Studies have been published that report the existence of bulges, i.e. local increases of the diameter, of MNTs [14] [15] [16] [17] [6] [18] [19] [20] [21] [22]. This ultrastructural feature of MNTs was attributed to the presence of objects (vesicles or organelles) inside MNTs [6].

\section{Objective}

Our objective was to document and analyze bulges of MNTs using scanning electron microscopy (SEM) to increase the knowledge of ultrastructural feature of MNTs. To our knowledge, a detailed characterization of MNT bulges concerning their geometrical 
properties has not been done before.
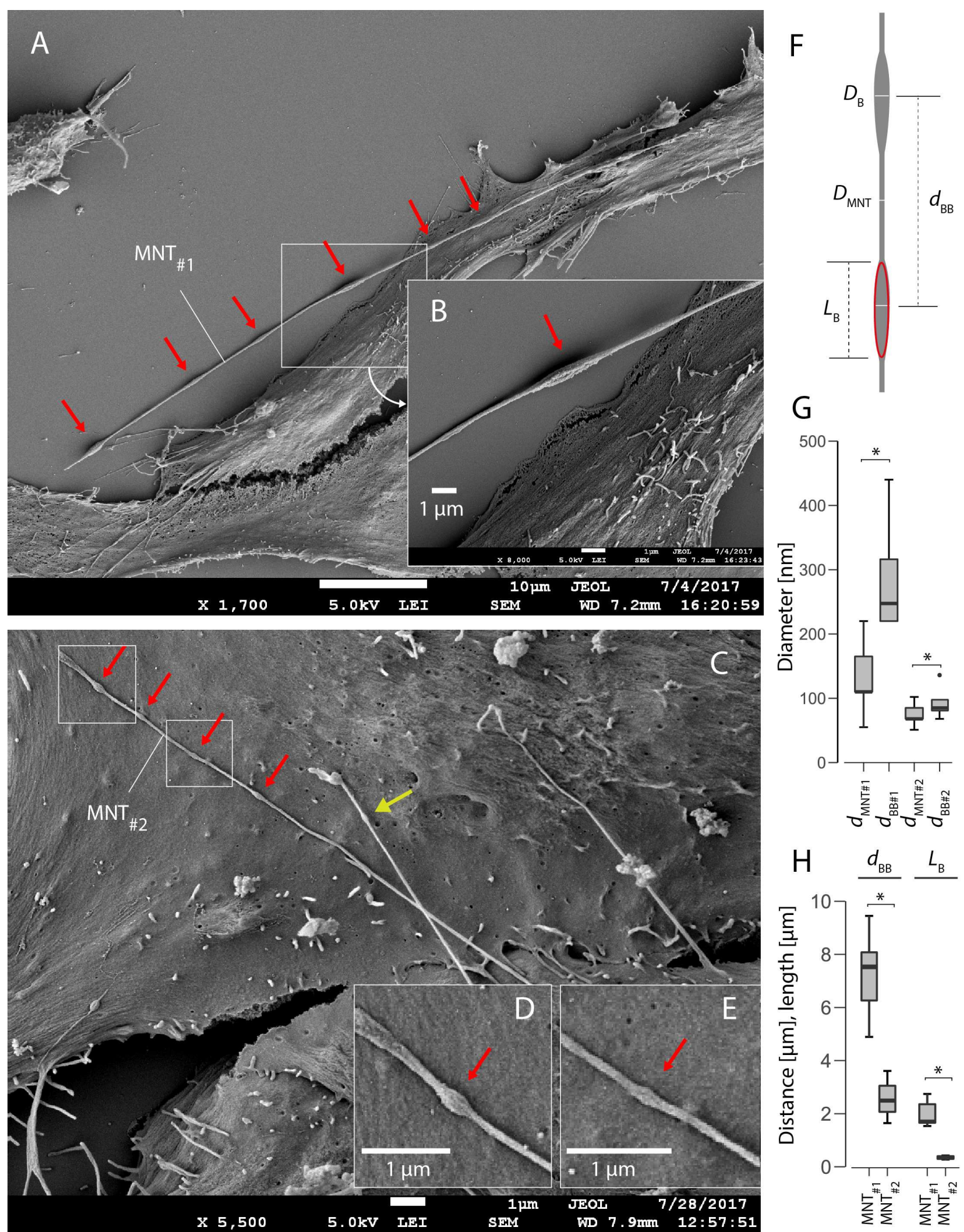

Figure Legend

Figure 1. Scanning electron microscopy (in lower secondary electron image mode) images of bulges of MNT between cone-like photoreceptor cells $(661 \mathrm{~W})$. (A, C) Two MNTs (MNT ${ }_{\# 1}$ and $\mathrm{MNT}_{\#_{2}}$ ) with bulges (indicated with red arrows). Scale bar of image A: $10 \mu \mathrm{m}$. Scale bar of image $\mathbf{C}: 1 \mu \mathrm{m}$. In figure $\mathbf{C}$, a single bulge of a third 
MNT is indicated with a yellow arrow. The two MNTs are connected at one side with the cell and on the other side unconnected. The disconnection seems to be the result of the SEM staining process, possibly disrupting the state of the MNTs causing the breakage of long MNTs in some cases.

(B) Magnification of a MNT bulge selected from image A.

(D, E) Magnification of two MNT bulges selected from image C. In $\mathbf{D}$ the bulge is clearly visible where in $\mathbf{E}$ it is more difficult to recognize the bulge.

(F) Diagrammatic drawing of an MNT with two bulges and the four parameters determined for further analysis: MNT diameter $\left(D_{\mathrm{MNT}}\right)$, diameter of the bulge $\left(D_{\mathrm{B}}\right)$, distance between adjacent bulges $\left(d_{\mathrm{BB}}\right)$ and the length of the bulges $\left(L_{\mathrm{B}}\right)$. In the lower visualized MNT bulge in $\mathbf{F}$ an oval is shown (red) that was used to determine $L_{\mathrm{B}}$.

$(\mathbf{G}, \mathbf{H})$ MNT diameter and bulge diameter values, as well as, distance between adjacent bulges for both MNTs. Statistically significant (Bayer factor $>10$, Bayesian independent samples $t$-test) differences are indicated $\left({ }^{*}\right)$.

It should be noted that $\mathrm{MNT}_{\# 1}$ is approximately twice as wide as $\mathrm{MNT}_{\# 2}$. Smaller cargo in $\mathrm{MNT}_{\# 1}$ might therefore not be visible.

\section{Results \& Discussion}

By analyzing cell cultures of the cone-like photoreceptor cell line $661 \mathrm{~W}$ via SEM, we found two MNTs $\left(\mathrm{MNT}_{\# 1}\right.$ and $\left.\mathrm{MNT}_{\# 2}\right)$ that clearly showed multiple bulges along their axis (see Fig. 1A-E). $\mathrm{MNT}_{\# 1}$ had a total length of $61.60 \mu \mathrm{m}$ and $\mathrm{MNT}_{\# 2}$ of $13.74 \mu \mathrm{m}$.

The MNT bulge diameter $\left(D_{\mathrm{B}}\right)$ was statistically significant larger for both MNTs compared to the MNTs diameters $\left(D_{\mathrm{MNT}}\right)$ determined on bulge-free parts of the MNTs (median, 95\% confidence interval $(\mathrm{CI})) ; D_{\mathrm{B}{ }_{1}}=247.5(220.0,440.0) \mathrm{nm}(n=6)$ vs. $D_{\mathrm{MNTH}}$ $=110.0(55.0,220.0) \mathrm{nm}(n=100)$, Bayes factor $(\mathrm{BF})>100$, effects size (Cohen's $d): d=$ $-4.389 ; \mathrm{D}_{\mathrm{B} \# 2}=85.0(68.0,136.0) \mathrm{nm}(n=4)$ vs. $D_{\mathrm{MNT \# 2}}=68.0(51.0,102.0) \mathrm{nm}(n=100)$, $\mathrm{BF}=23.4, d=1.609$ ) (see Fig. $1 \mathrm{G}$ ). This analysis proofs that the visually observed bulges are real deformations of the MNT membrane. The analysis also shows that the larger the diameter of the MNTs is, the larger is the diameter of the MNT bulges (as quantified by the larger absolute differences of the medians $(\Delta m)$ of DB values of $\operatorname{MNT}_{\# 1}(\Delta m=$ $137.5 \mathrm{~nm}$, increase of $225 \%)$ compared to $\mathrm{MNT}_{\# 2}(\Delta m=17.0 \mathrm{~nm}$, increase of $125 \%)$ ).

The distance between successive MNT bulges $\left(d_{\mathrm{BB}}\right)$ was $d_{\mathrm{BB}}=7.527(4.890,9.451) \mu \mathrm{m}$ for $\mathrm{MNT}_{\#_{1}}(n=5)$ and $d_{\mathrm{BB}}=2.492(1.644,3.610) \mu \mathrm{m}$ for $\mathrm{MNT}_{\#_{2}}(n=3)$. The difference was statistically significant $(\mathrm{BF}=11.27, d=3.041)$ (see Fig. $1 \mathrm{H})$.

The length of the MNT bulges $\left(L_{\mathrm{B}}\right)$ was $L_{\mathrm{B}}=1.700(1.536,2.742) \mu \mathrm{m}$ for $\mathrm{MNT}_{\#_{1}}(n=6)$ and $L_{\mathrm{B}}=0.3495(0.2730,0.4260) \mu \mathrm{m}$ for $\mathrm{MNT}_{\# 2}(n=4)$. The difference was statistically significant $(\mathrm{BF}=95.83, d=3.879$ ) (see Fig. $1 \mathrm{H})$.

Our finding of the presence of bulges on MNTs is in agreement with previously published studies with other cell types [14] [15] [6] [20]. Wittig et al. [6] documented examples of MNT bulges in the retinal pigment epithelial cells using differential interference contrast microscopy and SEM. The MNT bulge diameter was reported to be $D_{\mathrm{B}}$ $=1 \mu \mathrm{m}$ and the typical MNT diameter to be $D_{\mathrm{MNT}}=250 \mathrm{~nm}$ (with a range of $50-300$ $\mathrm{nm}$ ). These values are larger than the values we determined in our sample and study, i.e. the diameter of the two MNTs investigated in our study were $2.3\left(\mathrm{MNT}_{\# 1}\right)$ and 3.6 $\left(\mathrm{MNT}_{\#_{2}}\right)$ times smaller, and the bulge diameters were $4.04\left(\mathrm{MNT}_{\# 1}\right)$ and $11.75\left(\mathrm{MNT}_{\#_{2}}\right)$ times smaller, respectively. This indicates the presence of a large heterogeneity of MNT morphology, confirming previous reports. Wittig et al. concluded that the MNT bulges suggest the presence of organelles in MNTs. Using the specific mitochondrial dye JC-1 they could label mitochondria inside MNTs. The presence of mitochondria inside MNT bulges was also reported by Patheja and Sahu [22]. In another study, Reichert et al. [20] reported also the presence of MNT bulges between cells (human primary CD $34^{+}$ haematopoietic progenitor cells and leukaemic KG1a cells). The MNT bulge thickness was described to be smaller than $100 \mathrm{~nm}$ in diameter, in agreement with our finding concerning $\mathrm{MNT}_{\# 2}$.

With our study, we were the first to analyze $L_{\mathrm{B}}$ and $d_{\mathrm{BB}}$ values of MNT bulges. The range of $L_{\mathrm{B}}$ values obtained $\left(0.2730-2.74^{2} \mu \mathrm{m}\right)$ overlaps with the length distribution of mitochondria in cells (e.g. in retinal pigment epithelial ARPE-19 cells [23]: $0.4^{-74}$ $\mu \mathrm{m}$, primary cortical neurons [24]: $1.27 \pm 0.04 \mu \mathrm{m}$ ) as well as with the size distribution 
of peroxisomes $(0.1-1 \mu \mathrm{m})$ [25]. The length of mitochondria is, however, a variable parameter that depends not only on the specific cell type but also on the state of the cell, i.e. cell cycle [26] or metabolic state [27].

That the location of MNT bulges was seemingly not random on the MNT is a novel finding of our study. The distance values between successive MNT bulges, as quantified by $d_{\mathrm{BB}}$, showed unimodal distributions for both MNTs investigated. The ratio $d_{\mathrm{BB}} / L_{\mathrm{B}}$ based on the median values was 4.43 for $\mathrm{MNT}_{\#_{1}}$ and 7.13 for $\mathrm{MNT}_{\#_{2}}$, respectively; and the ratio $d_{\mathrm{BB}} / D_{\mathrm{B}}$ based on the median values was 68.4 for $\mathrm{MNT}_{\#_{1}}$ and 25 for $\mathrm{MNT}_{\#_{2}}$, respectively. This indicates that the periodicity of the bulges on an MNT seems to be independent of the MNT diameter as well as the length of the MNT bulges. Concerning the cause of the MNT bulge periodicity, no clear mechanism can be envisaged. Either it is a finding due to chance, or there is an underlying unexplored process that regulates the distance between successive bulges of MNTs. It could be indeed that it is only a finding due to chance since only a limited number of data points (i.e. distance values) were available for the analysis. Further studies are needed to investigate this aspect with a larger sample size.

What could be in general the cause of the MNT bulges observed? We think that three possible causes should be considered: (1) MNT bulges as artefacts caused by the cell staining process involving the SEM analysis, (2) MNT bulges as local deformations of the MNT without the presence of a cargo inside, or (3) MNT bulges as effects of an object inside the MNT that deforms the enclosing MNT locally.

Explanation (1) is unlikely since MNT bulges were also observed by optical microscopy [6], and MNT bulges are only occasionally observed (contrary to the expectation of the MNT bulge formation being a results of a general SEM staining artefact that should affect all or a large part of all MNTs present in the investigated culture). Explanation (2) is in principle possible but there are no nanomechanical processes known yet that result in an oval local deformation of an MNT without the involvement of an object inside the MNT causing the deformation. Explanation (3) is most likely since (i) the presence of mitochondria inside MNT bulges was previously shown by several studies [28] [17] [6] [29] [30] [18] [19] [22]; (ii) the MNT bulge diameter values determined in our study (range: $68.0-440.0 \mathrm{~nm}$ ) correspond to diameters of organelles like mitochondria (typical diameter: $100-1000 \mathrm{~nm}$ ) [31] [32] [33], peroxisomes (typical diameter in RPE cells: $100-300 \mathrm{~nm}$ ) [34], or exosomes (typical diameter: 30-100 $\mathrm{nm}$ ) [35] [36]; (iii) the length of the MNT bulges overlap with the length distributions of mitochondria and peroxisomes [23] [34], and (iv) movements of MNT bulges were reported by other studies (speed: $0.16 \mu \mathrm{m} / \mathrm{s}$ [14], $20.7 \pm 2.3 \mu \mathrm{m} / \mathrm{h}$ [37], $0.08 \mu \mathrm{m} / \mathrm{s}$ [19], $0.0259 \pm 0.0079 \mu \mathrm{m} / \mathrm{s}$

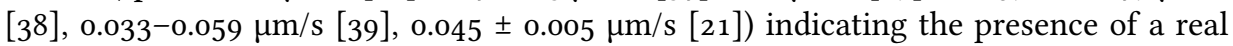
object inside the MNT.

\section{Conclusions}

Our SEM analysis of two MNTs from cone-like photoreceptor cells of the cell line 661W with multiple bulges revealed that (i) bulges are characterized by a statistically significant local increase in MNT diameter (125\% and $225 \%$ for both MNTs investigated); (ii) the thickness and length of the MNT bulges correspond to dimensions of mitochondria, peroxisomes and exosomes, and (iii) the MNT bulges seem not to be randomly distributed on the MNTs but exhibit preferred spacing with a different median value for each MNT. Our findings shed new light on the microstructure of MNTs and MNT bulges in particular.

Further studies should replicate and extend our findings with more MNTs and by applying SEM and fluorescence microscopy simultaneously by correlative light and electron microscopy (CLEM) [4o] or even better 3D CLEM with serial blockface (SBF) SEM, as recently developed [41].

\section{Limitations}

There are two main limitations in this study. First, only two MNTs could be identified in the SEM images taken from multiple sections of two cell cultures, resulting in a small number of MNT bulges that could be used for the statistical analysis of their 
geometrical properties. Secondly, we did not employ fluorescence microscopy to a nalyzewhether the MNT bulges were colocalized with the presence of fluorescence markers, e.g. JC-1 indicating the mitochondria. This, however, could not be performed for technical reasons: performing SEM and fluorescence microscopy analysis of the same MNT consecutively with two instruments (as available in our lab) is nearly impossible due to change and disturbance of the cell/MNT state caused by the transportation between microscopes and the time-delay involved processing the sample for SEM analysis causing changes in the MNT state. A CLEM analysis would have been a solution.

\title{
Additional Information
}

\author{
Methods and Supplementary Material \\ Please see https://sciencematters.io/articles/201802000011.
}

\section{Acknowledgements}

The author would like to thank Dr. Thomas Kurth (TU Dresden, Center for Regenerative Therapies Dresden) for realization of electron microscopy and Simon Schaefer for proofreading the manuscript.

\section{Ethics Statement}

Not applicable.

\section{Citations}

[1] Gerdes et al. "Tunneling nanotubes: A new route for the exchange of components between animal cells". In: FEBS Letters 581.11 (2007), pp. 2194-2201

[2] Hans-Hermann Gerdes and Rainer Pepperkok. "Cell-to-cell communication: current views and future perspectives". In: Cell and Tissue Research 352.1 (2013), pp. 1-3

[3] Ariazi et al. "Tunneling Nanotubes and Gap Junctions-Their Role in Long-Range Intercellular Communication during Development, Health, and Disease Conditions". In: Frontiers in Molecular Neuroscience 10 (2017), p. 333.

[4] Hans-Hermann Gerdes and Raquel Negrão Carvalho. "Intercellular transfer mediated by tunneling nanotubes". In: Current Opinion in Cell Biology 20.4 (2008), pp. 470-475.

[5] Marzo et al. "Multifaceted Roles of Tunneling Nanotubes in Intercellular Communication”. In: Frontiers in Physiology 3 (2012), p. 72 .

[6] Wittig et al. "Multi-Level Communication of Human Retinal Pigment Epithelial Cells via Tunneling Nanotubes”. In: PLOS ONE $7 \cdot 3$ (2012), e33195.

[7] Zhang et al. "Membrane nanotubes: Novel communication between distant cells”. In: Science China Life Sciences 56.11 (2013), pp. 994-999.

[8] McCoy-Simandle et al. "Exosomes and nanotubes: Control of immune cell communication". In: The International fournal of Biochemistry and Cell Biology 71 (2016), pp. 44-54.

[9] Austefjord et al. "Tunneling nanotubes". In: Communicative and Integrative Biology 7.1 (2014), e27934.

[10] Spafford et al. "Developing Neurons Form Transient Nanotubes Facilitating Electrical Coupling and Calcium Signaling with Distant Astrocytes". In: PLOS ONE 7.10 (2012), e47429.

[11] Amin Rustom. "The missing link: does tunnelling nanotube-based supercellularity provide a new understanding of chronic and lifestyle diseases?” In: Open Biology 6.6 (2016), p. 160057.

[12] Felix Scholkmann. "Long range physical cell-to-cell signalling via mitochondria inside membrane nanotubes: a hypothesis". In: Theoretical Biology and Medical Modelling 13.1 (2016), p. 16.
[13] Felix Scholkmann. "Two emerging topics regarding long-range physical signaling in neurosystems: Membrane nanotubes and electromagnetic fields". In: fournal of Integrative Neuroscience 14.02 (2015), pp. 135-153.

[14] Björn Önfelt et al. "Cutting Edge: Membrane Nanotubes Connect Immune Cells”. In: The fournal of Immunology 173.3 (2004), pp. $1511-1513$.

[15] Holly R. Chinnery, Eric Pearlman, and Paul G. McMenamin. "Cutting Edge: Membrane Nanotubes In Vivo: A Feature of MHC Class II+ Cells in the Mouse Cornea". In: The fournal of Immunology 180.9 (2008), pp. 5779-5783.

[16] X Sun et al. "Tunneling-nanotube direction determination in neurons and astrocytes". In: Cell Death and Disease 3.12 (2012), e438.

[17] Krishna C. Vallabhaneni, Hermann Haller, and Inna Dumler. "Vascular Smooth Muscle Cells Initiate Proliferation of Mesenchymal Stem Cells by Mitochondrial Transfer via Tunneling Nanotubes". In: Stem Cells and Development 21.17 (2012), pp. 3104-3113

[18] Kaiming Liu et al. "Mesenchymal stem cells rescue injured endothelial cells in an in vitro ischemia-reperfusion model via tunneling nanotube like structure-mediated mitochondrial transfer”. In: Microvascular Research 92 (2014), pp. 10-18.

[19] X Wang and H-H Gerdes. "Transfer of mitochondria via tunneling nanotubes rescues apoptotic PC12 cells". In: Cell Death and Differentiation 22.7 (2015), pp. 1181-1191.

[20] Doreen Reichert et al. "Tunneling nanotubes mediate the transfer of stem cell marker CD133 between hematopoietic progenitor cells". In: Experimental Hematology 44.11 (2016), 1092-1112.e2.

[21] Ian Parker et al. "Lattice light sheet imaging of membrane nanotubes between human breast cancer cells in culture and in brain metastases”. In: Scientific Reports 7.1 (2017), p. 11029.

[22] Pooja Patheja and Khageswar Sahu. "Macrophage conditioned medium induced cellular network formation in $\mathrm{MCF}-7$ cells through enhanced tunneling nanotube formation and tunneling nanotube mediated release of viable cytoplasmic fragments". In: Experimental Cell Research 355.2 (2017), pp. 182-193. 
[23] Bantseev et al. "Mitochondrial "Movement" and Lens Optics following Oxidative Stress from UV-B Irradiation”. In: Annals of the New York Academy of Sciences 1091.1 (2006), pp. 17-33.

[24] Zanelli et al. "Nitric oxide impairs mitochondrial movement in cortical neurons during hypoxia". In: Journal of Neurochemistry 97.3 (2006), pp. 724-736.

[25] Jennifer J. Smith and John D. Aitchison. "Peroxisomes take shape". In: Nature Reviews Molecular Cell Biology 14.12 (2013), pp. 803-817.

[26] Kennady et al. "Variation of mitochondrial size during the cell cycle: A multiparameter flow cytometric and microscopic study". In: Cytometry Part A 62A.2 (2004), pp. 97-108.

[27] Timothy Wai and Thomas Langer. "Mitochondrial Dynamics and Metabolic Regulation”. In: Trends in Endocrinology and Metabolism 27.2 (2016), pp. 105-117.

[28] Acquistapace et al. "Human Mesenchymal Stem Cells Reprogram Adult Cardiomyocytes Toward a Progenitor-Like State Through Partial Cell Fusion and Mitochondria Transfer". In: STEM CELLS 29.5 (2011), pp. 812-824.

[29] Jennifer Pasquier et al. "Preferential transfer of mitochondria from endothelial to cancer cells through tunneling nanotubes modulates chemoresistance". In: fournal of Translational Medicine 11.1 (2013), p. 94.

[30] Xiang Li et al. "Mitochondrial Transfer of Induced Pluripotent Stem Cell-Derived Mesenchymal Stem Cells to Airway Epithelial Cells Attenuates Cigarette Smoke- Induced Damage". In: American Journal of Respiratory Cell and Molecular Biology 51.3 (2014), pp. 455-465.

[31] Gregson et al. "A Comparative Study of Brain and Liver Mitochondria from New-Born and Adult Rats". In: fournal of Neurochemistry 16.4 (1969), pp. 617-626.
[32] Ilja Bobylev et al. "Depletion of Mitofusin-2 Causes Mitochondrial Damage in Cisplatin-Induced Neuropathy”. In: Molecular Neurobiology 55 (2018), pp. 1227-1235.

[33] Kolossov et al. "Airyscan super-resolution microscopy of mitochondrial morphology and dynamics in living tumor cells". In: Microscopy Research and Technique 81 (2018), pp. 115-128.

[34] Janos Feher et al. "Mitochondrial alterations of retinal pigment epithelium in age-related macular degeneration”. In: Neurobiology of Aging 27.7 (2006), pp. 983-993.

[35] Clotilde Théry, Laurence Zitvogel, and Sebastian Amigorena. "Exosomes: composition, biogenesis and function". In: Nature Reviews Immunology 2 (2002), pp. 569-579.

[36] Jin et al. "Imaging of exosomes by broadband scanning microwave microscopy”. In: 201646 th European Microwave Conference (EuMC) (2016), pp. 1211-1214.

[37] Tavi et al. "Myogenic skeletal muscle satellite cells communicate by tunnelling nanotubes". In: Journal of Cellular Physiology 223 (2010), pp. 376-383

[38] Amin Rustom et al. "Nanotubular Highways for Intercellular Organelle Transport”. In: Science 303.5660 (2004), pp. 1007-1010.

[39] Bénard et al. "Structural and functional analysis of tunneling nanotubes (TnTs) using gCW STED and gconfocal approaches". In: Biology of the Cell 107.11 (2015), pp. 419-425.

[40] Minoo Razi and Sharon A. Tooze. "Chapter 17 Correlative Light and Electron Microscopy”. In: Autophagy in Mammalian Systems and Part B: Methods in Enzymology 452 (2009), pp. 261-275.

[41] Matthew R. G. Russell et al. " 3 D correlative light and electron microscopy of cultured cells using serial blockface scanning electron microscopy". In: Journal of Cell Science 130.1 (2017) pp. $278-291$. 